\title{
An innovative low-profile monolithic constant-temperature anemometer
}

\author{
David M. Birch ${ }^{\mathrm{a}}$, Jonathan F. Morrison ${ }^{\mathrm{b}}$ \\ ${ }^{a}$ Faculty of Engineering and Physical Sciences, University of Surrey, U.K. \\ ${ }^{b}$ Department of Aeronautics, Imperial College London, U.K.
}

\begin{abstract}
A constant-temperature anemometer has been developed which uses a single highfidelity speaker driver as a combined signal and power amplifier. Owing to its small size and simplicity of construction, the anemometer is well suited for applications requiring a large number of channels (such as hot-wire rakes) as well as applications requiring the embedding of instrumentation within confined experimental models (such as reduced-scale wind turbine blades). The anemometer is shown to have performance characteristics similar to those of a commercial anemometer when used under its design conditions. An operating bandwidth as high as $10 \mathrm{kHz}$ can be achieved, which is greater than most available time-resolved digital particle-image velocimetry systems and is shown to be sufficient to track large-scale turbulence structures in channel flow.
\end{abstract}

Key words:

Constant-temperature anemometry, hot-wire, instrumentation

\section{Introduction}

Constant-temperature anemometry is generally accepted as a well-developed and mature technique for the measurement of turbulent flows: see, for example, 
the reviews of Comte-Bellot (1976), Goldstein (1983), Hayward (1979), Hinze (1975), Perry (1982), Perry and Morrison (1971), Richards (1977) and Smoliakov (1983), to list but a few. High-specification multi-channel constant-temperature anemometer (CTA) units are currently available commercially. However, for certain particular experimental applications, the use of these commercial anemometers is not appropriate. For example, measurements within the rotating frame of a model wind turbine would require the anemometer to be mounted within the rotor; in this case, commercial anemometers would be too large and heavy. Also, measurements within very large wind tunnels often require very long signal cables, thereby increasing the contamination of experimental data by electromagnetic interference. Mounting a large commercial anemometer within the wind tunnel to reduce the cable length is not normally practicable because so doing would result in a large amount of blockage and interference. In addition, commercially available CTA systems may not be ideal for applications requiring a very large number of sensor channels (such as surface-mounted, thin-film sensor arrays or high-resolution hot-wire rakes), owing to the very high per-channel cost of these commercial systems.

For these sorts of applications, bespoke anemometer circuits are often constructed 'in-house', and a number of extensively validated classical CTA circuit designs have been published (Itsweire and Helland, 1983; Janssen et al., 1959; Miller et al., 1987; Wyngaard and Lumley, 1967). However, the majority of the classical CTA circuits available in the literature were developed more than thirty years ago, so the design of these circuits was constrained by the limited availability and variety of high-precision, high-current semiconductor devices. Any new anemometer based on these designs will therefore be unnecessarily complex, and 
would not be taking full advantage of the dramatic advances in semiconductor technology which have since been made.

While some recent efforts have been dedicated to the application of new semiconductor technology to the development of simple CTAs, the emphasis has either been on the integration of the sensor with the electronic components in a single monolithic package for industrial applications (Mayer et al., 1997b,a; Fang et al., 2007), or on the micro-electromechanical manufacturing of sensing elements by means of novel techniques (Chen and Chang, 2003; Ebefors et al., 1998; Nagaiah et al., 1987). Owing to the fairly low demand for laboratory CTA systems, it is unlikely that a purpose-designed monolithic integrated CTA circuit suitable for turbulent flow measurement will become commercially available. However, the adaptation of a common off-the-shelf component for use as a simple CTA would provide the benefits of a monolithic circuit (such as a very small package outline, low noise susceptibility and simple construction) without requiring complex semiconductor photolithography facilities.

\section{Monolithic CTA Circuit design}

High-fidelity speaker drivers are almost ideally suited for the purposes of constant temperature anemometry, as they are high-precision devices which combine signal and power amplification in a single integrated circuit package. Because they are intended for use in audio devices, the operating bandwidth of these amplifiers will typically be equivalent to the range of human hearing $(20 \mathrm{kHz})$, which is sufficiently high for many laboratory-scale turbulent flow measurement applications. With the addition of a small number of external passive components, a very simple but functional CTA can be constructed. 


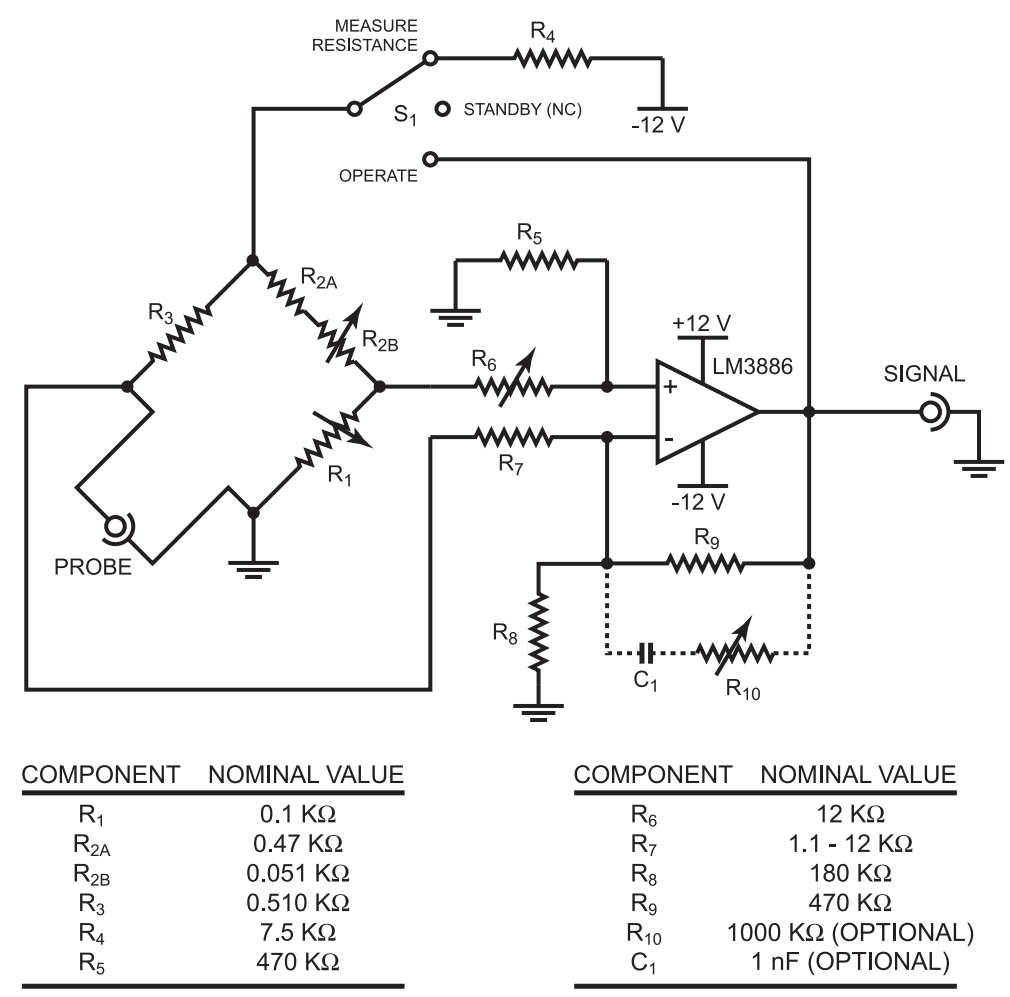

Figure 1: Schematic representation of the monolithic CTA circuit. 
Figure 1 shows a schematic representation of the present monolithic audio speaker-driver constant-temperature anemometer (monolithic CTA). A National Semiconductor LM3886 audio power amplifier is used to drive a Wheatstone bridge with a resistance ratio of 10:1 (trimmed via the potentiometer $R_{2 B}$ ), allowing the probe resistance and overheat ratio to be read and set directly and with reasonable accuracy using a graduated turn-counting knob on the potentiometer $R_{1}$. A bridge ratio as high as 10:1 has already been shown to have a marginal effect on the performance of a classical CTA (Itsweire and Helland, 1983). The resistances $R_{6}$ and $R_{7}$, nominally between 1 and $22 \mathrm{k} \Omega$, are tuned and selected, respectively, to compensate for the significant unit-to-unit variation in the zero-input offset voltage of the LM3886. The amplifier feedback filter network is required in order to eliminate a high-frequency pole which may reduce the usable bandwidth, and is tuned to achieve the best frequency response for each individual amplifier. The filter network could be omitted in order to further reduce the complexity of the circuit, though this will reduce the effective bandwidth. The values of $C_{1}$ and $R_{10}$ are typically of the order of $10^{-9} \mathrm{~F}$ and $10^{6} \Omega$, respectively. A simplified analytical model of the circuit suggests that the low-pass roll-off due to the feedback filter is negligible relative to parasitic effects. While the imbalances in the resistances associated with the amplifier inputs introduce an uncertainty in the measurement of the probe resistance and in the setting of the overheat ratio, the resulting error is typically less than $10 \%$, and may be minimized by trimming $R_{2 B}$. While this uncertainty does not affect the performance of the anemometer, temperature correction schemes requiring precise knowledge of the overheat ratio would transfer this uncertainty to the velocity measurements in post-processing and should not be used with this CTA. 
The mode of operation of the monolithic CTA is controlled via the threeposition switch $S_{1}$. With $S_{1}$ in the "measure resistance" position, the bridge is powered by a constant voltage, allowing the bridge to be used to determine the probe resistance. With $S_{1}$ in the "standby" position, the bridge is unpowered, protecting the probe from potentially hazardous transients which may occur when the amplifier power is switched on or off, or when the probe is removed or reconnected. When $S_{1}$ is in the "operate" position, the bridge is powered by the amplifier and the feedback loop is completed.

Because the anemometer consists of a single integrated circuit component, the circuitry may be constructed to occupy an exceptionally small volume. The required circuitry may be assembled using only $26 \times 36 \mathrm{~mm}$ of standard 2.54 mm-pitch electronic strip-board (excluding the foot-prints of the balancing potentiometer $R_{1}$ and mode selector switch $S_{1}$, to which the user will require frequent access). Owing to the low cost of the LM3886 amplifier (less than GB $£ 4$ at the time of printing) and the ease with which the circuit may be assembled (approximately 50 solder points including terminal connections, depending on the layout), this CTA is suitable for applications where a very large number of channels is required: see, for example, Tutkun et al. (2009).

When used in the audio applications for which it was designed, the LM3886 exhibits a typical slew rate of $19 \mathrm{~V} / \mu \mathrm{s}$, a gain-bandwidth product of $8 \mathrm{MHz}$ and a maximum error of $0.03 \%$ at frequencies up to $20 \mathrm{kHz}$ (National Semiconductor, 2003). However, because of the significant differences in the driven loads and the large output DC offsets (the manufacturer warns that decoupling capacitances must be used on the amplifier output), the performance of the amplifier is expected to deviate from the published nominal standards when it is used to drive a hot- 
wire bridge. The off-design performance specifications of the LM3886 are not known and the component architecture is not available to the user. Therefore the design of the monolithic CTA circuit was refined through a process of trial and error. The accurate modelling of the coupled thermal-electric system based upon the published component specifications is also precluded, and an extensive experimental validation has been carried out in order to establish empirically the static and dynamic performance characteristics and limitations of the circuit.

\section{CTA Validation}

Measurements were carried out in a rough-wall turbulent channel flow. The flow facility consists of a rectangular channel with half-height $h=51 \mathrm{~mm}$, width $W=15 h$ and length $L=132 h$. The top and bottom wetted surfaces of the channel are lined with an open-type, 16-grit heavy industrial abrasive (with a mean particle diameter of $k \sim 2.0 \mathrm{~mm}$ and sparse distribution) to ensure well-developed, fully-rough turbulent flow conditions at the measurement station. All of the results used for validation were collected at a distance $x / h=128$ from the channel inlet, at a wall-normal distance of $y=h$, unless otherwise noted. The fluid mean velocity was monitored at the $(x / h, y / h) \supseteq(128,1)$ measurement station using a Pitot tube. Further details of the experimental apparatus, as well as of the validation of the flow facility and the technique used for determining the friction velocity, are available in a related paper (Birch and Morrison, 2011).

The anemometer was used to drive a single 5- $\mu \mathrm{m}$-diameter silver-plated platinum hot-wire probe (Dantec model 55P01) with an etched sensing length of approximately $0.5 \mathrm{~mm}$, and connected to the bridge by means of a $2 \mathrm{~m}$ length of standard BNC cable. No compensation in the monolithic CTA bridge circuitry 
has been made for the cable capacitance or inductance. Sensor cables with other lengths varying from $1 \mathrm{~m}$ to $2.5 \mathrm{~m}$ were also tested, and no significant variation in performance was observed within the sensor bandwidth. The anemometer overheat ratio was set to a nominal value of 0.8 in all cases, and the fluid temperature was constant to within $0.5^{\circ} \mathrm{C}$ during each of the measurements. No corrections for fluid temperature change or sensor length were applied, and no analogue linearization was implemented. Signals were sampled for 70 seconds at $60 \mathrm{kHz}$, and were digitized using a Data Translation DT9836 16-bit data acquisition system with a full-scale range of $20 \mathrm{~V}$. The sampling rate was deliberately set much higher than the anticipated time-response of the CTA in order to adequately characterize the high-frequency roll-off. The CTA signals acquired for spectral analysis were offset, amplified with a nominal gain factor of 10, and then low-pass RC filtered at $100 \mathrm{kHz}$ (yielding a gain of 0.98 and 0.86 at $20 \mathrm{kHz}$ and $60 \mathrm{kHz}$, respectively) to suppress very-high-frequency signal noise prior to digitization at $60 \mathrm{kHz}$. For the purposes of comparison, all measurements were repeated using a commercial DISA model 55M10 anemometer with a tuned bridge stability compensator to drive the same sensor. The commercial anemometer system is known to have a frequency response of greater than $40 \mathrm{kHz}$ (for the range of mean velocities used here) and this is therefore taken to be sufficient to validate the response of the monolithic CTA. Signals from both CTAs were conditioned, acquired and processed identically, so any systemic errors or aliasing effects would not affect the comparison.

Figure 2 shows a typical calibration curve for the monolithic CTA, with the signal taken directly from the bridge top. It should be noted that, for shielding purposes, the bridge was configured such that the output signal is always nega- 

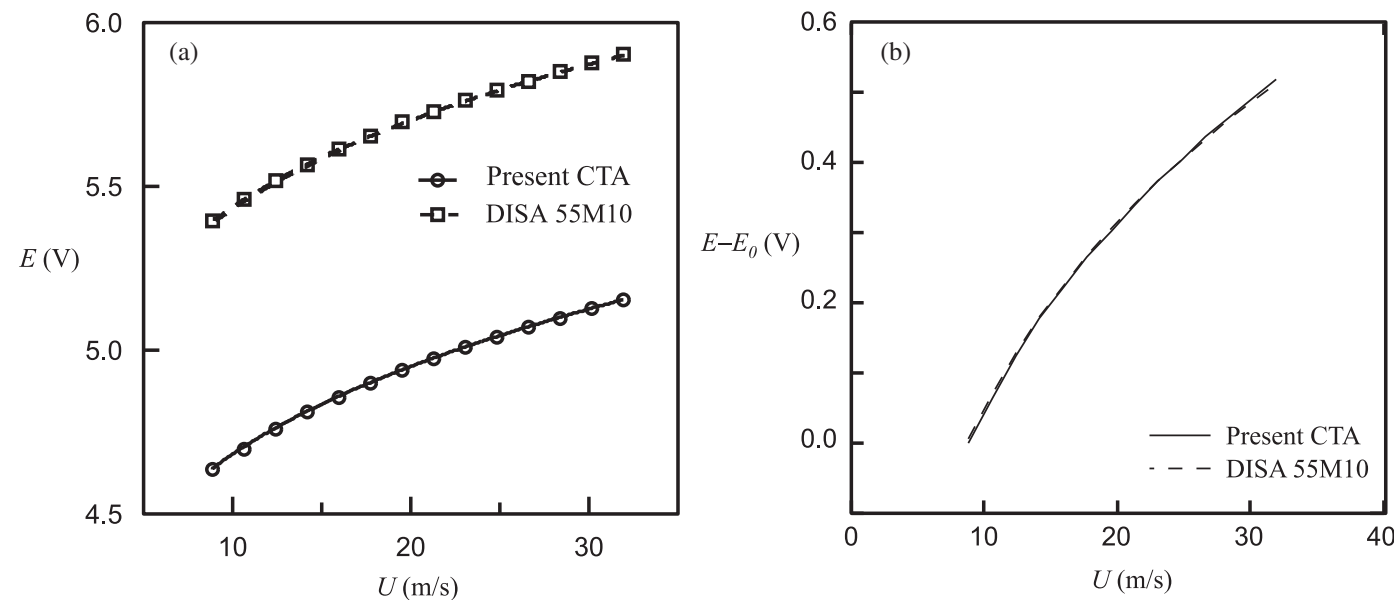

Figure 2: Typical CTA calibration curves. (a) Comparison of relative signal magnitudes, and (b) offset to show similarity. Note that the sign of the signal from the monolithic CTA has been numerically reversed in post-processing.

tive and decreases with increasing velocity. For the purposes of comparison, the signals from the monolithic CTA were numerically inverted in post-processing. Though the probe was calibrated in situ using the time-average of a fluctuating nonlinear signal, the error stemming from the linear approximation was estimated according to the method of Breuer (1995). Typical results are shown in Table 1, where the error in the mean velocity and the second and third velocity moments is shown for calibrations of order $n=2,3$ and 4. For all of the present results, $n=3$. Table 1 also shows the statistical convergence error $\delta$ for data records on the channel centreline. The convergence error in all cases is less than the typical experimental uncertainty, and for the means and second moments, is at least an order of magnitude smaller.

To further validate the function of the monolithic CTA, results were compared to the published rough-wall channel flow data of Bakken et al. (2005), which 


\begin{tabular}{lccc}
\hline$n$ & \multicolumn{3}{c}{ Errors } \\
& $\mathrm{U}$ & $\overline{u^{2}}$ & $\overline{u^{3}}$ \\
\hline 2 & $0.17 \%$ & $0.34 \%$ & $2.8 \%$ \\
3 & $0.14 \%$ & $0.38 \%$ & $1.4 \%$ \\
4 & $0.15 \%$ & $0.63 \%$ & $8.1 \%$ \\
\hline$\delta$ (CTA) & $0.063 \%$ & $0.27 \%$ & $4.6 \%$ \\
$\delta$ (DISA) & $0.015 \%$ & $0.25 \%$ & $3.3 \%$ \\
\hline
\end{tabular}

Table 1: Maximum errors in determining the velocity moments, estimated using the method of Breuer (1995) for the cases of calibrations of order $n=2,3$ and 4, together with convergence errors for $n=3$ for both the monolithic CTA and the DISA 55M10.

were collected at similar roughness Reynolds numbers. Figure 3 shows the wallnormal profiles of inner- and outer-scaled mean velocity, as well as ${\overline{u^{2}}}^{+}$and ${\overline{u^{3}}}^{+}$. The inner-scaled mean velocity shows a small but constant displacement between the present results and those of Bakken et al. (2005), which is consistent with the usual roughness-dependent offset. The roughness-independent outer-scaled mean velocity agrees very well with the previous data set. A difference in the outer-scaled second moment is apparent, however this appears to be due to the unexplained bifurcation in the data of Bakken et al. (2005); comparison against a different case (typical of their other observed mode) shows good agreement through to the edge of the roughness sublayer. The outer-scaled third moment also agrees reasonably well, given the typical experimental uncertainty inherent in higher-order velocity moments. A more extensive comparison of the results from the monolithic CTA to this and other existing data sets is available in Birch and Morrison (2011).

The frequency response of the CTA was evaluated first by means of the stan- 

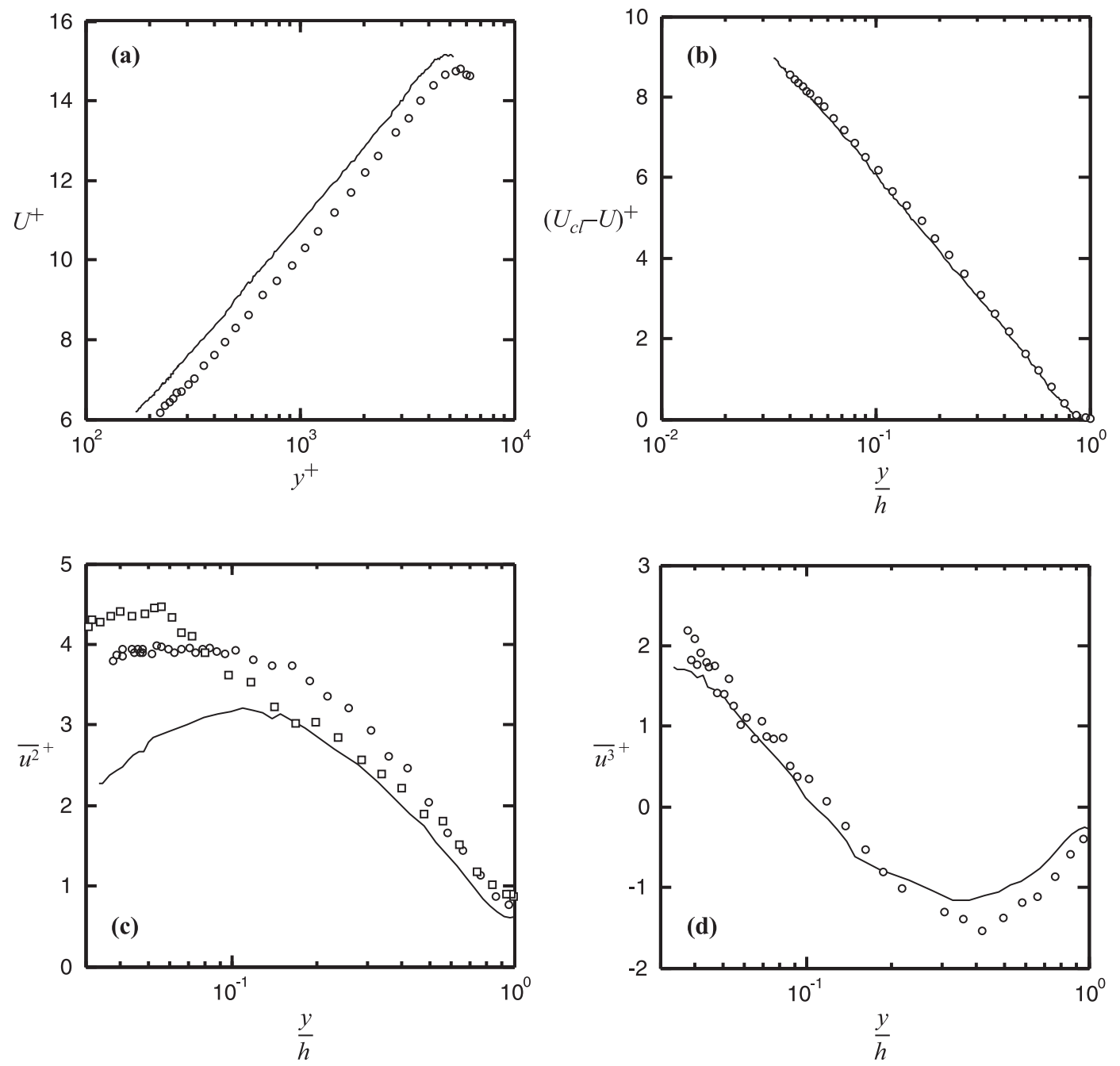

Figure 3: Comparison against the results of Bakken et al. (2005). (a), Inner-scaled mean velocity; (b), outer-scaled mean velocity; (c), second moment and (d) third moment. ——, present results, $R e_{\tau}=5130$; ○, Bakken et al. (2005), $R e_{\tau}=5600$; $\square$, Bakken et al. (2005), $R e_{\tau}=450$ (reproduced from Birch and Morrison, 2011). 


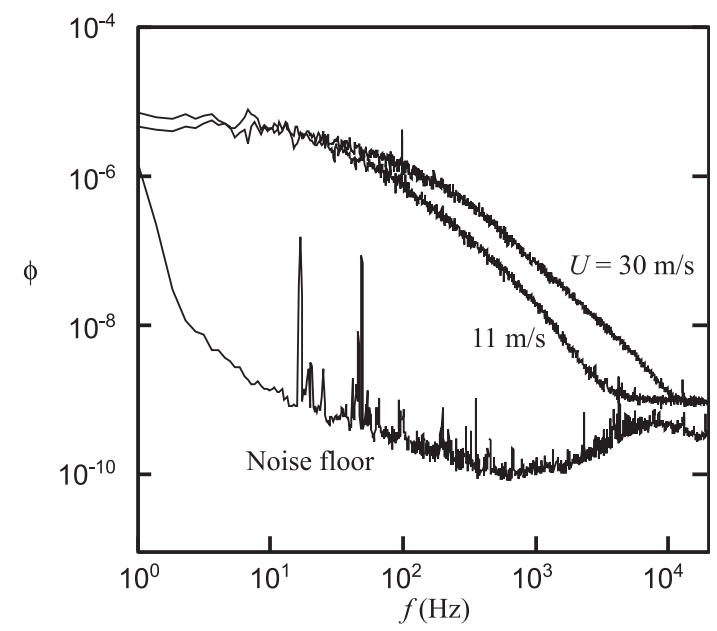

Figure 4: Signal power spectrum of CTA noise, compared to typical measurement signals at $y / h=$ 1.

dard square wave test, in which the circuit is impulsively perturbed from its steady state, and the resulting damped oscillations of the amplifier output are recorded. The monolithic CTA was tested with the sensor in quiescent air, as this set point yielded the slowest response. A typical overdamped response was observed, and a $97 \%$ signal recovery was achieved in a critical time of approximately $110 \mu \mathrm{s}$. Applying the approximation of Freymuth (1977), the minimum cut-off frequency of the anemometer is approximately $7 \mathrm{kHz}$. The signal slew rate was also measured, and found to be at least $2.06 \times 10^{4} \mathrm{~V} / \mathrm{s}$, corresponding to a velocity gradient of $1.67 \times 10^{6} \mathrm{~m} / \mathrm{s}^{2}$.

The zero-signal noise floor of the anemometer was also investigated. Figure 4 shows the power spectrum of the signal when the sensor was subjected to no flow, as well as the range of (uncalibrated) power spectra from experimental measurements. Through most of the bandwidth, the signals were nearly four decades higher than the noise floor. 

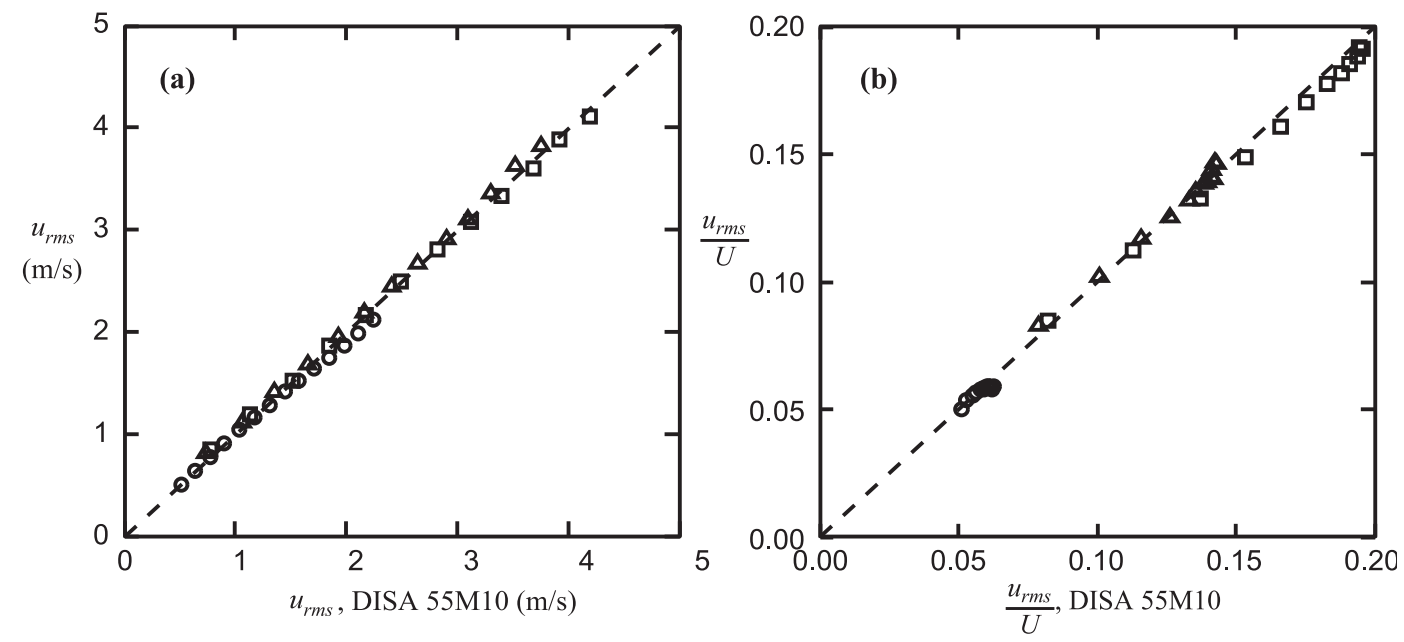

Figure 5: Comparison between the standard deviation of the velocity signals obtained with the present monolithic CTA and the DISA 55M10. (a) Dimensional values, and (b) normalized values. $\circ: y / h=1 ; \triangle: y / h=0.2 ; \square: y / h=0.1$.

In order to evaluate the performance of the monolithic CTA in typical laboratoryscale turbulent flows, signals from a single, typical CTA unit were collected at three wall-normal locations within the channel with 14 mean centreline flow speeds ranging from 9 to $32 \mathrm{~m} / \mathrm{s}$ using a single-wire probe. Figure 5 compares the standard deviation of the velocity signals to those obtained using the commercial system. For the case of lower mean flow speeds ( $U \lesssim 20 \mathrm{~m} / \mathrm{s}$ ), the two agree fairly well, after which the monolithic CTA begins to roll off as a higher proportion of the fluctuations occur at frequencies greater than its bandwidth.

The frequency response is further illustrated by the power spectra, which are shown in Figure 6 for three different local mean velocities at $y / h=1$ and 0.1 (thereby illustrating a range of mean velocities and turbulence intensities). Note that the spectra have been offset for clarity. For the case of $y / h=1$ (characterized by fairly low turbulence intensity), at $U=11 \mathrm{~m} / \mathrm{s}$, the power spectrum from 

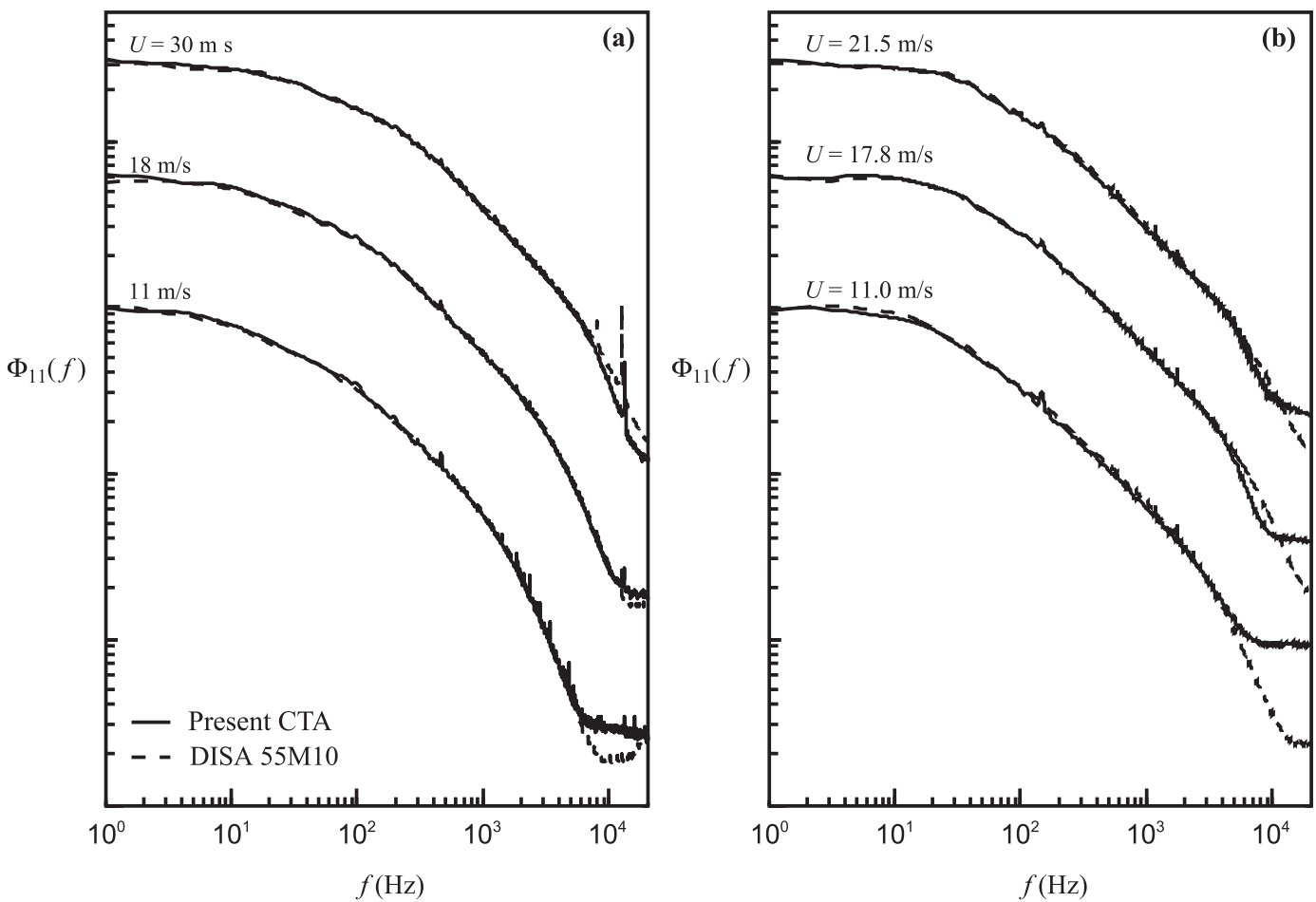

Figure 6: Comparison between the normalized velocity power spectra obtained using the monolithic CTA and the DISA 55M10. Note that the curves have been offset for clarity. (a) $y / h=1$; (b) $y / h=0.1$. 

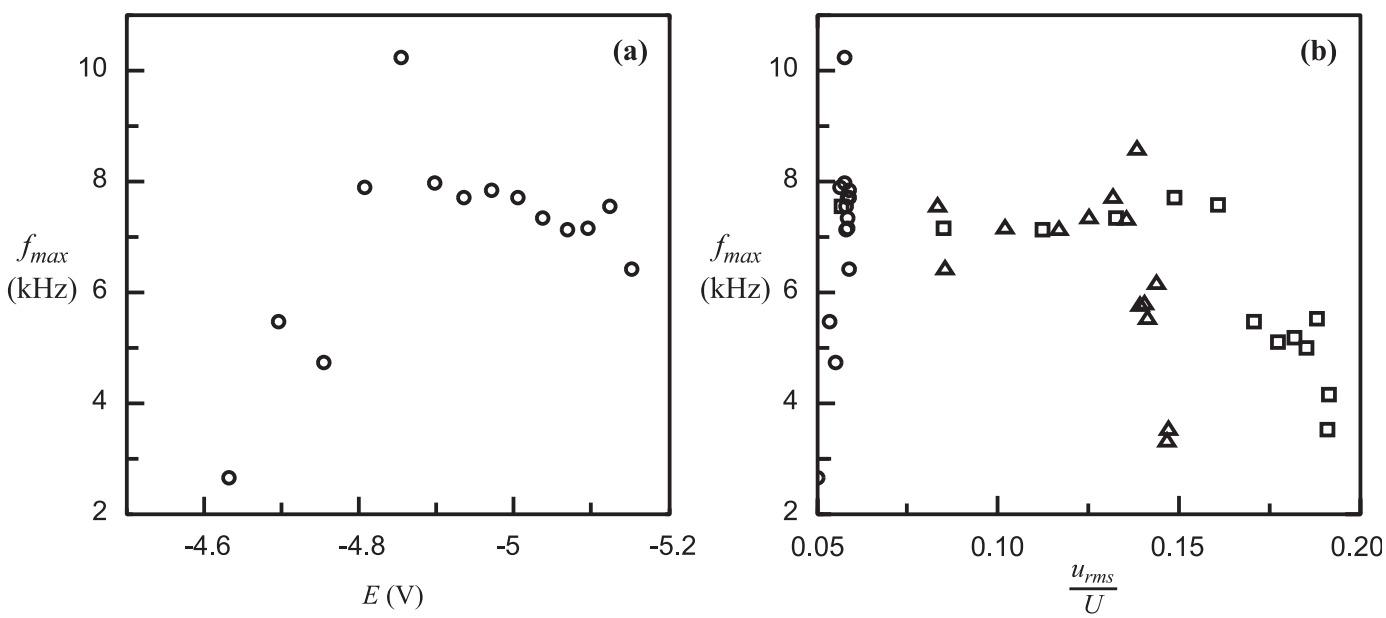

Figure 7: Variation of the roll-off frequency with amplifier set-point for the present monolithic CTA. (a) Variation with circuit set point; (b) Sensitivity to turbulence intensity with varying $U$. $\circ: y / h=1 ; \triangle: y / h=0.2 ; \square: y / h=0.1$.

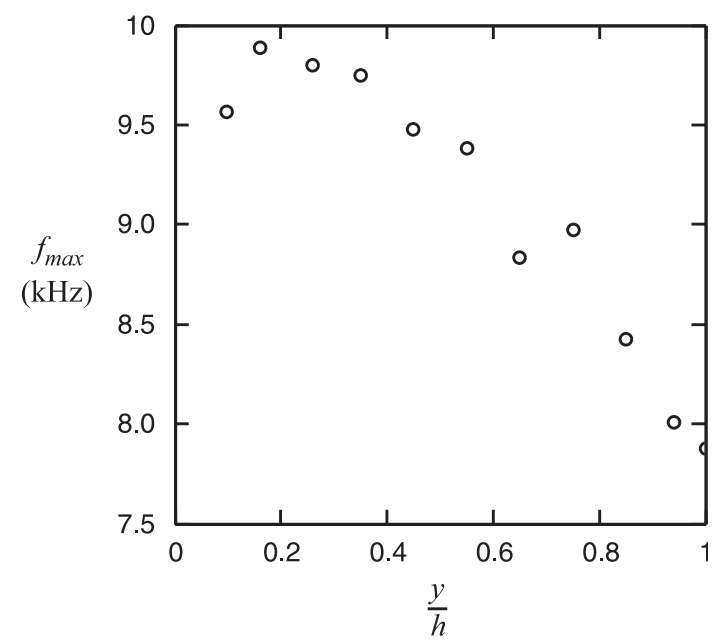

Figure 8: Variation of the roll-off frequency with wall-normal distance for the present monolithic CTA at the design centreline mean velocity of $26.6 \mathrm{~m} / \mathrm{s}$. 
the monolithic CTA coincides with that of the commercial system up to approximately $5.5 \mathrm{kHz}$, as the small-amplitude fluctuations at this low speed yield a low signal-to-noise ratio. At $U=18 \mathrm{~m} / \mathrm{s}$, the spectra agree well up to approximately $10 \mathrm{kHz}$. At $U=30 \mathrm{~m} / \mathrm{s}$, the monolithic CTA begins to roll off at $7 \mathrm{kHz}$, illustrating how the frequency response of the circuit varies with the set point. When the turbulence intensity is increased by a factor of about 5 , the higher-energy fluctuations further accentuate the limitations of the frequency response of the monolithic CTA, though this remains close to the cutoff frequency of about $6 \mathrm{kHz}$ through most of the mean velocity range. For the cases of lower turbulence intensity, The frequency $f_{\max }$ at which the monolithic CTA begins to roll off was computed and is plotted against the CTA bridge voltage in Figure 7 (a). As the bridge excitation increases from $-4.63 \mathrm{~V}$ to $-4.85 \mathrm{~V}, f_{\max }$ increases rapidly; this effect is attributed more to the increasing of the signal-to-noise ratio than the response of the CTA. The frequency response achieves a maximum around $E=-4.85 \mathrm{~V}$, and then begins to decrease with further increases in $E$. This is probably because the LM3886 amplifier was not designed to be operated with high output DC offsets. Figure 7 (b) shows $f_{\max }$ as a function of $u_{r m s} / U$ through a wide range of operating conditions and set points. The frequency response was insensitive to the turbulence intensity through most of the operating range, though $f_{\max }$ tended to decrease once the set point of the circuit had increased sufficiently beyond the optimal range. Figure 7 (b) therefore suggests that the monolithic CTA should not be used to measure flows of high turbulence intensity $\left(\sqrt{\overline{u^{2}}} / U \gtrsim 0.15\right)$ when the mean flow speed is sufficiently removed from the design set-point.

Table 2 summarizes the bandwidth limitations of the monolithic CTA. Data sets were collected over a range of wall-normal distances and mean centreline 


\begin{tabular}{|c|c|c|c|c|}
\hline \multirow[t]{2}{*}{$U(\mathrm{~m} / \mathrm{s})$} & \multicolumn{3}{|c|}{$\sqrt{\overline{u^{2}}} / U$} & \multirow[t]{2}{*}{$f_{\max }(\mathrm{kHz})$} \\
\hline & DISA & & & \\
\hline 26.7 & 0.0617 & 0.0580 & $(-6.1)$ & 7.2 \\
\hline 26.1 & 0.145 & 0.148 & $(2.4)$ & 9.6 \\
\hline 16.0 & 0.0578 & 0.0574 & $(-0.6)$ & 3.6 \\
\hline 16.3 & 0.134 & 0.133 & $(-0.8)$ & 8.0 \\
\hline 10.6 & 0.0529 & 0.0532 & $(0.6)$ & 3.2 \\
\hline 9.0 & 0.163 & 0.166 & (1.8) & 6.2 \\
\hline
\end{tabular}

Table 2: Turbulence intensity and bandwidth of the monolithic CTA, obtained by comparison to the DISA 55M10. Values in parentheses are percent differences.

velocities achievable with the flow facility. Data records showing similar mean velocities but with very different turbulence intensities were then tabulated, enabling the limiting cases of low mean velocity and high turbulence intensity (and likewise, cases of high mean velocity and low turbulence intensity) to be directly compared. At relatively high mean velocities of $U \sim 26 \mathrm{~m} / \mathrm{s}$, bandwidths of over $7 \mathrm{kHz}$ are obtained for turbulence intensities ranging from $6.2 \%$ through $14.5 \%$. A larger difference in the second velocity moments is observed for the low-turbulence case, though, owing to the relatively large amplitude of the highfrequency noise. As the mean velocity decreases (changing the set-point of the system), the sensitivity of the unit to high-frequency fluctuations decreases with the signal-to-noise ratio. In the worst case (low speed, low turbulence intensity), a bandwidth of only $3.2 \mathrm{kHz}$ was obtained- however, it should be noted that, under these measurement conditions, the bandwidth of the commercial CTA was likewise reduced.

As a demonstration of the bandwidth limitations encountered during typical 
rough-wall channel measurements, Figure 8 shows the variation of $f_{\max }$ as a function of wall-normal distance at the design channel centreline velocity of $26 \mathrm{~m} / \mathrm{s}$. The bandwidth increases with increasing wall proximity, as a result of the increasing signal-to-noise ratio. The smallest effective bandwidth of $f_{\max } \sim 7.8 \mathrm{kHz}$ is obtained at the channel centreline, where the signal-to-nose ratio is lowest.

Too few channels have yet been constructed to adequately assess the range of unit-to-unit variability in the optimal set point, though the differences in the set-points of the fourteen channels constructed so far are of the same order as the uncertainty in the overheat ratio. The frequency response may always be optimized for particular applications by a careful selection of the overheat ratio and etched sensing length of the probe, which determine the set-point of the circuit.

While the frequency response of the monolithic CTA is substantially lower than that of a commercial system, it should be noted that the bandwidth within the turbulence measurable with a hot-wire is limited by the spatial resolution of the sensor, and may be orders of magnitude lower. For example, a typical sensor of length $l=1 \mathrm{~mm}$ used to measure a turbulent flow with a mean velocity $U$ of 10 $\mathrm{m} / \mathrm{s}$ has a maximum resolvable frequency of $U / l=10 \mathrm{kHz}$ only.

\section{Hot-wire rake results}

In order to assess the usefulness of the monolithic CTAs in tracking the large structures within wall-bounded turbulence, a rake of hot-wire sensors was used to examine rough-wall channel flow. The very-large, quasi-streamwise structures within wall-bounded turbulence have been of considerable recent interest owing to their importance in the dynamics of the near-wall turbulence cycle (Davenport, 1961; Kim and Adrian, 1999; Hunt and Morrison, 2000), and since the 
length scales of the structures within the flow may span several orders of magnitude, they may not all be resolved simultaneously by near-wall particle-image velocimetry (PIV) measurements (Ganapathisubramani et al., 2003; Tomkins and Adrian, 2003). Consequently, rakes of hot wires continue to be used to track these large structures within wall layers, often using Taylor's "frozen-flow" approximation (Ganapathisubramani et al., 2005; Hutchins and Marusic, 2007; Monty et al., 2007; Tutkun et al., 2009). Rakes have also been instrumental in a number of studies investigating the fundamental nature of turbulence (Swearingen and Blackwelder, 1987; Glezer et al., 1989; Bonnet et al., 1998; Metzger and Klewicki, 2001). However, Dennis and Nickels (2008) have recently shown how significant differences between fields evolving in space and those evolving in time are due primarily to the small-scale motion. Therefore data obtained from measurements using a rake of hot wires continue to supplement those available from PIV, and may even complement PIV measurements directly (see, for example, the work of Coudert et al., 2011).

A rake of 12 sensors with a spacing of $5.08 \mathrm{~mm}$ was constructed in a manner similar to that described by Monty et al. (2007). Rake data were sampled simultaneously at $4 \mathrm{kHz}$, and a total of $2^{21}$ samples were collected per channel. The statistical convergence error remained insignificant relative to the overall experimental uncertainty. Measurements were made at a channel centre-line mean velocity $U$ $=26.6 \mathrm{~m} / \mathrm{s}$, corresponding to a Reynolds number $R e_{\tau}=h u_{\tau} / v=5130$ (where $u_{\tau}$ is the friction velocity and $v$ is the kinematic viscosity) and a non-dimensional roughness $k^{+}=k u_{\tau} / v=200$ (where $k$ is the mean roughness height). Here, the $x, y$ and $z$ axes are oriented along the streamwise, wall-normal and spanwise directions, respectively. 


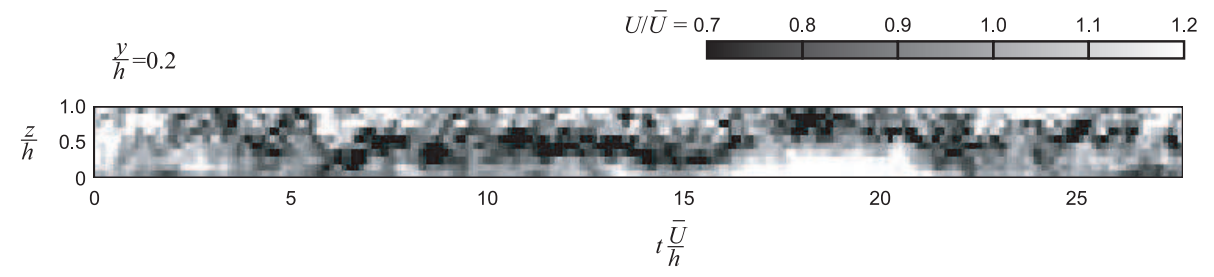

Figure 9: Illustration of simultaneous time-domain velocity measurements collected with the hotwire rake at $y_{0} / h \approx 0.3$

Figure 9 shows the streamwise mean velocity recorded by the rake sensors as a function of time. Over the dimensionless time-interval of $0<t U / h<25$, there is a streamwise pattern of alternating low- and high-velocity regions at a wallnormal distance $y_{0} / h \sim 0.3$. The strength, streamwise persistence and spanwise periodicity of these structures are similar to those observed in boundary layers by Hutchins and Marusic (2007) and in internal flows by Monty et al. (2007).

The two-point longitudinal velocity correlation,

$$
R_{11}=\frac{\overline{u\left(y_{0}, z_{0} ; t\right) u\left(y_{0}, z_{0}+\Delta z ; t+\Delta t\right)}}{\overline{u\left(y_{0}, z_{0}\right)^{2}}},
$$

(where the subscript ' 0 ' denotes the position of the fixed sensor, and $\Delta z$ is the spanwise separation) was also measured using the rake results. At $y_{0} / h \approx 0.3$ (Figure 10), a large region of negative correlation is observed with a peak at $\Delta z / h \sim 0.7$, which is consistent with the spanwise extent of the structures observed in Figure 9 and the limitation of Taylor's hypothesis proposed by Dennis and Nickels (2008). Figure 11 shows $R_{11}(\Delta z, \Delta t)$ at $y_{0} / h \approx 0.69$ : at this height, the region of negative correlation has more or less disappeared, suggesting that this is the outer limit to the large-scale quasi-streamwise structures.

The ability of the monolithic CTA to resolve the structures apparent in Figure 9 was quantitatively validated by comparing two-point spanwise correlations of 


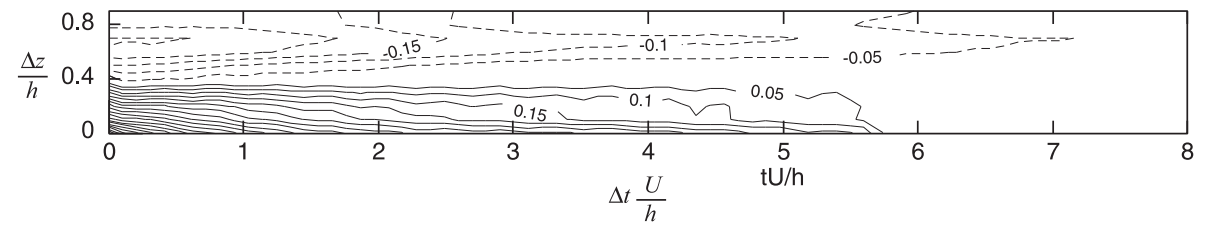

Figure 10: Two-point longitudinal correlation as a function of $\Delta z / h$ and $\Delta t U / h$ at $y_{0} / h \approx 0.3$. Dashed lines indicate $R_{11}<0$.

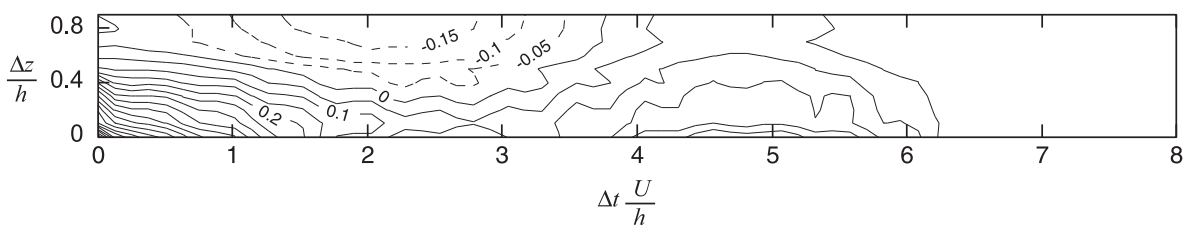

Figure 11: Two-point longitudinal correlation as a function of $\Delta z / h$ and $\Delta t U / h$ at $y_{0} / h \approx 0.69$ Dashed lines indicate $R_{11}<0$.

the streamwise velocity to those reported in previous studies of internal flows. A good agreement is achieved between the present results and the collapsed pipeand channel-flow data of Monty et al. (2007) for $600 \lesssim R e_{\tau} \lesssim 3000$ (Figure 12).

\section{Conclusions}

A highly simplified constant-temperature anemometer has been developed using an adapted off-the-shelf high-fidelity speaker driver as a combined signal and power amplifier. The resulting unit is simple to construct and tune, and may be constructed to occupy a very small volume. The unit is therefore an attractive alternative for use in applications where the anemometer must be embedded in an experimental model (such as in the rotating frame of a wind turbine rotor) or when a very large number of channels is required. The static sensitivity of the anemometer is similar to that of a commercial unit. The frequency response varies with the mean bridge voltage, but was shown to typically range between $6 \mathrm{kHz}$ and $10 \mathrm{kHz}$ 


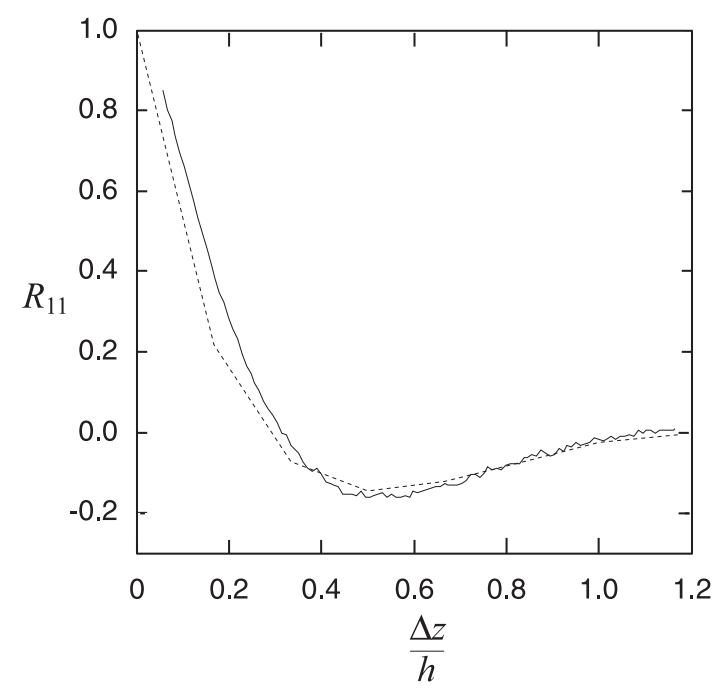

Figure 12: Two-point spanwise correlation at $y / h \sim 0.15$. — data of Monty et al. (2007) (reproduced from Birch and Morrison, 2011).

when the CTA is used under its design conditions, which is an improvement upon most digital PIV systems. The use of the anemometer was also demonstrated together with a hot-wire rake by tracking and characterizing the large streamwise coherent structures in rough-wall channel flow.

\section{Acknowledgments}

This work was supported by the Engineering and Physical Sciences Research Council of Great Britain under grant number EP/D037166.

\section{References}

Bakken, O. M., Krogstad, P. A., Ashrafian, A., Andersson, H. I., 2005. Reynolds number effects in the outer layer of the turbulent flow in a channel with rough walls. Phys. Fluids 17, 065101. 
Birch, D. M., Morrison, J. F., 2011. Similarity of the streamwise velocity component in very-rough-wall channel flow. Journal of Fluid Mechanics 668, 174201.

Bonnet, J. P., Delville, J., Glauser, M. N., Antonia, R. A., Bisset, D. K., Cole, D. R., Fiedler, H. E., Garem, J. H., Hilberg, D., Jeong, J., Kevlahan, N. K. R., Ukeiley, L. S., Vincendeau, E., 1998. Collaborative testing of eddy structure identification methods in free turbulent shear flows. Exp. Fluids 25 (3), 197225.

Breuer, K. S., 1995. Stochastic calibration of sensors in turbulent flow fields. Exp. Fluids 19 (2), 138-141.

Chen, J., Chang, L., 2003. Development and characterization of surface micromachined, out-of-plane hot-wire anemometer. J. Microelectromech. Systems 12 (6), 979-988.

Comte-Bellot, G., 1976. Hot-wire anemometry. Ann. Rev. Fluid Mech. 8, 209231.

Coudert, S., Foucaut, J. M., Kostas, J., Stanislas, M., Braud, P., Fourment, C., Delville, J., Tutkun, M., Mehdi, F., Johansson, P., George, W. K., 2011. Double large field stereoscopic piv in a high reynolds number turbulent boundary layer. Exp. Fluids 50 (1), 1-12.

Davenport, A. G., 1961. The spectrum of horizontal gustiness near the ground in high winds. Q. J. Roy. Meteor. Soc. 87, 194-211.

Dennis, D. J., Nickels, T. B., 2008. On the limitations of Taylor's hypothesis in 
constructing long structures in a turbulent boundary layer. J. Fluid Mech. 614, 197-206.

Ebefors, T., Kalvesten, E., Stemme, G., 1998. Three dimensional silicon triplehot-wire anemometer based on polyimide joints. In: Proceedings of the 11th Annual International Workshop on Micro Electro Mechanical Systems. pp. 9398.

Fang, Y., Chen, J., Lee, H., Yen, K., Wang, C., 2007. Commercialized cmos compatible micro anemometer. In: Proceedings of the 5th IEEE Conference on Sensors. pp. 1452-1455.

Freymuth, P., 1977. Frequency response and electronic testing for constanttemperature hot-wire anemometers. J. Phys. E: Sci. Instr. 10 (7), 705-710.

Ganapathisubramani, B., Hutchins, N., Hambleton, W. T., Longmire, K. E., Marusic, I., 2005. Investigation of large-scale coherence in a turbulent boundary layer using two-point correlations. J. Fluid Mech. 524, 57-80.

Ganapathisubramani, B., Longmire, E. K., Marusic, I., 2003. Characteristics of vortex packets in turbulent boundary layers. J. Fluid Mech. 478, 35-46.

Glezer, A., Kadioglu, Z., Pearlstein, A. J., 1989. Development of extended proper orthogonal decomposition and its application to a time periodically forced plane mixing layer. Phys. Fluids A 1 (8), 1363-1373.

Goldstein, R., 1983. Fluid Mechanics Measurements. Hemisphere.

Hayward, A., 1979. Flow meters: A Basic Guide and Source Book for Users. MacMillan. 
Hinze, J., 1975. Turbulence, 2nd Ed. McGraw-Hill.

Hunt, J. C. R., Morrison, J. F., 2000. Eddy structure in turbulent boundary layers. Eur. J. Mech. B - Fluids 19, 673-694.

Hutchins, N., Marusic, I., 2007. Evidence of very long meandering features in the logarithmic region of turbulent boundary layers. J. Fluid Mech. 579, 1-28.

Itsweire, E., Helland, K., 1983. A high-performance low-cost constanttemperature hot-wire anemometer. J. Phys. E: Sci. Instr. 16 (6), 549-553.

Janssen, J., Ensing, L., Erp, J. V., 1959. A constant-temperature-operation hotwire anemometer. Proc. IRE 47 (4), 555-567.

Kim, K. C., Adrian, R. J., 1999. Very large-scale motion in the outer layer. Phys. Fluids A 11, 417-422.

Mayer, F., Haberli, A., Jacobs, H., Ofner, G., Paul, O., Baltes, H., 1997a. Singlechip cmos anemometer. In: IEEE Technical Digest, International Electron Devices Meeting. pp. 895-898.

Mayer, F., Paul, O., Baltes, H., 1997b. Flip-chip packaging for thermal cmos anemometers. In: Proceedings of the IEEE 10th annual International Workshop on MEMS. pp. 203-208.

Metzger, M. M., Klewicki, J. C., 2001. A comparative study of near-wall turbulence in high and low reynolds number boundary layers. Phys. Fluids 13 (3), $692-701$.

Miller, I., D. Shah, D., Antonia, R., 1987. A constant temperature hot-wire anemometer. J. Phys. E: Sci. Instr. 20 (3), 311-314. 
Monty, J. P., Stewart, J. A., Williams, R. C., Chong, M. S., 2007. Large-scale features in turbulent pipe and channel flows. J. Fluid Mech. 589, 147-156.

Nagaiah, N., Sleiti, A., Rodriguez, S., Kapat, J., An, L., Chow, L., 1987. A novel design and analysis of a mems ceramic hot-wire anemometer for high temperature applications. J. Phys. Conf. Series 34, 277-282.

National Semiconductor, 2003. Lm3886 overture audio power amplifier series high-performance $68 \mathrm{w}$ audio power amplifier with mute. Product specification DS011833.

Perry, A., 1982. Hot-Wire Anemometry. Clarendon.

Perry, A., Morrison, G., 1971. A study of the constant-temperature hot-wire anemometer. J. Fluid Mech. 47 (3), 577-599.

Richards, B., 1977. Measurement of Unsteady Fluid Dynamic Phenomena. Hemisphere.

Smoliakov, A., 1983. The Measurement of Turbulent Fluctuations: an Introduction to Hot-Wire Anemometry and Related Transducers. Springer-Verlag.

Swearingen, J. D., Blackwelder, R. F., 1987. The growth and breakdown of streamwise vortices in the presence of a wall. J. Fluid Mech. 182 (8), 255-290.

Tomkins, C. D., Adrian, R. J., 2003. Spanwise structure and scale growth in turbulent boundary layers. J. Fluid Mech. 490, 37-74.

Tutkun, M., George, W. K., Delville, J., Stanislas, M., Johansson, P. B. V., Foucaut, J. M., Coudert, S., 2009. Two-point correlations in high reynolds number flat plate turbulent boundary layers. J. Turbulence 10 (21), 1-23. 
Wyngaard, J., Lumley, J., 1967. A constant temperature hot-wire anemometer. J. Sci. Instr. 44 (35), 363-365. 